\title{
Article \\ A statistical inference of the Principle of Maximum Entropy Production
}

\author{
Martijn Veening ${ }^{1,+} \mathbb{C}$
}

\author{
1 EntropoMetrics; martijn@entropometrics.com \\ + Current address: Electrastraat 21,9801 WD Zuidhorn, The Netherlands
}

\begin{abstract}
The maximization of entropy $S$ within a closed system is accepted as an inevitability (as the second law of thermodynamics) by statistical inference alone. The Maximum Entropy Production Principle (MEPP) states that not only $S$ maximizes, but $\dot{S}$ as well: a system will dissipate as fast as possible. There is still no consensus on the general validity of this MEPP, even though it shows remarkable explanatory power (both qualitatively and quantitatively), and has been empirically demonstrated for many domains. In this theoretical paper I provide a generalization of entropy gradients, to show that the MEPP actually follows from the same statistical inference, as that of the 2nd law of thermodynamics. For this generalization I only use the concepts of super-statespaces and microstate-density. These concepts also allow for the abstraction of 'Self Organizing Criticality' to a bifurcating local difference in this density, and allow for a generalization of the fundamentally unresolved concepts of 'chaos' and 'order'.
\end{abstract}

Keywords: entropy production maximization, complexity, thermodynamics, nonlinear dynamics, statistical mechanics, autopoiesis, entropy, entropy production, life

\section{Introduction}

In the last few decades, several attempts have been made to explain the occurrence and stability of what we call 'living systems', or 'order out of chaos'. Most notably, the alleged conflict between 'autopoiesis' and the second law of thermodynamics is still unresolved [12]. Despite the impressive progress and multidisciplinary approaches in this field, there is still no conclusive consensus on any of these aspects [13].

This theoretical paper should provide this consensus, not by presenting yet another new theory to describe living systems, but rather by generalizing existing concepts to prove the so-called 'Maximum Entropy Production Principle', and then infer the occurrence and local stability of autopoiesis as a statistical possibility.

\subsection{Thermodynamic entropy}

The well-known Boltzmann equation for thermodynamic entropy in a closed system is:

$$
S=-k_{B} \ln W
$$

where $\mathrm{W}$ indicates the number of microstates that correspond to a certain macrostate. The well-known Second Law of Thermodynamics tells us that the state of a system will tend towards macrostates with a higher $W$. The trivial example of such a macrostate is a homogeneous distribution.

It's irreversibility comes from statistical inference: the state of a system has a higher probability to have a macrostate with many microstates, than a macrostate with little microstates. Therefore, over time, it's $W$ (and $S$ ) will increase up to some maximum. 


\subsection{Maximization of entropy-production}

Not only will a system maximize its entropy $S$ over time, it will always do so as fast as possible. This maximization of $\dot{S}$ is called the 'maximum entropy production principle' (MEPP) [1] [2]. This principle has been empirically validated for many domains, such as the atmosphere [11] [6] [9], crystallization [5], enzyme reactions [3] and the economy [4]. Intuitively this makes sense, but this principle is still debated, and its ubiquitous validity has not been demonstrated yet [10] [7] [8].

The materials and methods in this paper will provide for the generalizations necessary to finally infer this ubiquitous validity with the same logic as that of the second law of thermodynamics, and then statistically infer the occurrence of 'order'.

\section{Materials and Methods}

\subsection{Dynamic state-spaces and super-statespaces}

By convention we consider the state-space of a system having some dimensionality $n \in \mathbb{N}$, so we can describe the state of a system as some $w \in \mathbb{R}^{n}$. If we generalize to systems with a dynamic state-space (systems of which the statespace changes over time), then such a statespace itself has a statespace. We provide the following definition:

Definition 1. A super-statespace is the statespace of a statespace.

The simplest systems then have a zero-dimensional super-statespace with only a single solution, and more complex systems can have super-statespaces with many solutions.

An example of a system with a super-statespace with a single solution is the textbookexample of a thermodynamic closed system of some gas that dissipates energy.

Examples of systems with a dynamic state-space are complex, non-linear dynamic systems, such as living system, ecosystems, economies, technological systems and financial systems.

\subsection{Microstate density}

Entropy is quantified using the number of microstates of some macrostate. To illustrate the entropic gradient (from low to high entropy) one can use a heatmap for the number of microstates. Such a heatmap actually shows the 'microstate density' of a macrostate.

In this sense, the second law of thermodynamics can be illustrated by a trajectory of the state towards an area with a higher microstate-density. Figure 1 shows the entropic gradient of a system with a static statespace. It has a zero-dimensional super-statespace (with a single solution, and only a single-dimensional entropic gradient).

Figure 1. One-dimensional entropic gradient of a macrostate $W$

A dynamic state-space can contain areas where dimensionality of complexity increases (or decreases) over time. This means that in that region of the state-space, there are much more micro-states that correspond to the macrostate: another region with relative high microstate-density.

These areas are visualized in Figure 2. It clearly shows these 'waves' of micro-state density of a system with a non-zero-dimensional super-statespace. It shows that systems with non-zero-dimensional super-statespaces can have multidimensional entropic gradients. 


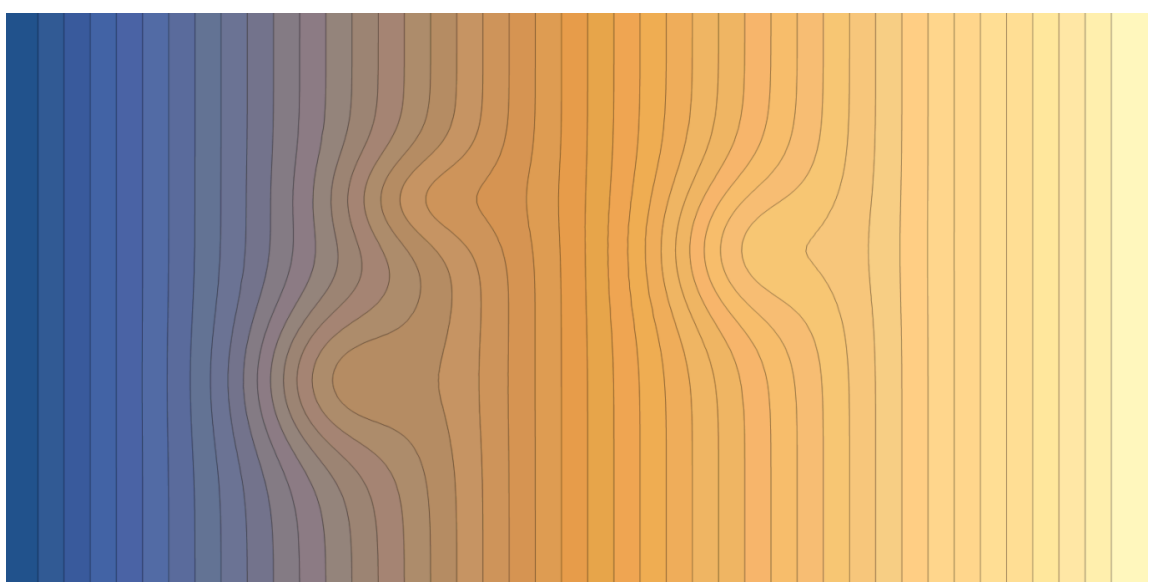

Figure 2. Heatmap of micro-state density with 2-dimensional entropic gradients

We have now generalized the entropic gradient of systems with a zero-dimensional super-statespace as a special case of multi-dimensional entropic gradients of systems with a super-statespace of any dimensionality (including none).

\subsection{The inevitability of entropy production maximization}

We have seen that complex dynamic systems can show multidimensional entropic gradients: either towards higher microstate-density within the same superstate (a static statespace), or towards superstates with a higher dimensional complexity. It then depends on which of these gradients is the steepest (relative to the initial/current state of the system), for the system to follow.

So the same statistical inference applies as with the second law of thermodynamics: if the state of a system enters a region with ascending dimensionality (more microstates), the state progresses on this ascent because it has the highest probability to tend towards the state with the most microstates available locally. By definition, this yields the highest amount of entropy production possible.

This shows the inevitability and ubiquitous validity of the maximization of entropy production .

\subsection{Self-organizing criticalities}

All kinds of external and internal dynamics can alter the state-space, as the state finds the way of maximum entropy production. For example, biochemical structures can collide and form more stable structures, allowing for more complex dynamics. Local maxima of entropy production can emerge and disappear.

These can mathematically be described as bifurcations from unstable to stable attractors (e.g. limit cycles) and vice versa, of non-linear entropy production dynamics. These local maxima, where $\ddot{S}=0$, are clearly visible in the heatmap in Figure 3 , which is only a more extreme version of Figure 2.

These local maxima are the sets of micro-states $\vec{x}$ which satisfy:

$$
\frac{\partial^{2} S(\vec{x}, t)}{\partial t^{2}}=0
$$

If such a local maximum emerges (and the state of a system is caught in its basin of attraction) we recognize this process as a self-organizing criticality. Eventually such a maximum will disappear again (because of local saturation/exhaustion of entropy production, roughly speaking), and this implies a collapse of the self-organizing criticality. For organic systems, this is called death.

The statespace then decreases in dimensionality again, and resumes its dissipative course, or gets caught in another basis of attraction.

In other words, these areas mark the emergence of what we call 'order', but from this 
generalization it is actually an acceleration towards more disorder (albeit local in space and time). Or rather: order and chaos are generalized into relative concepts (instead of discrete).

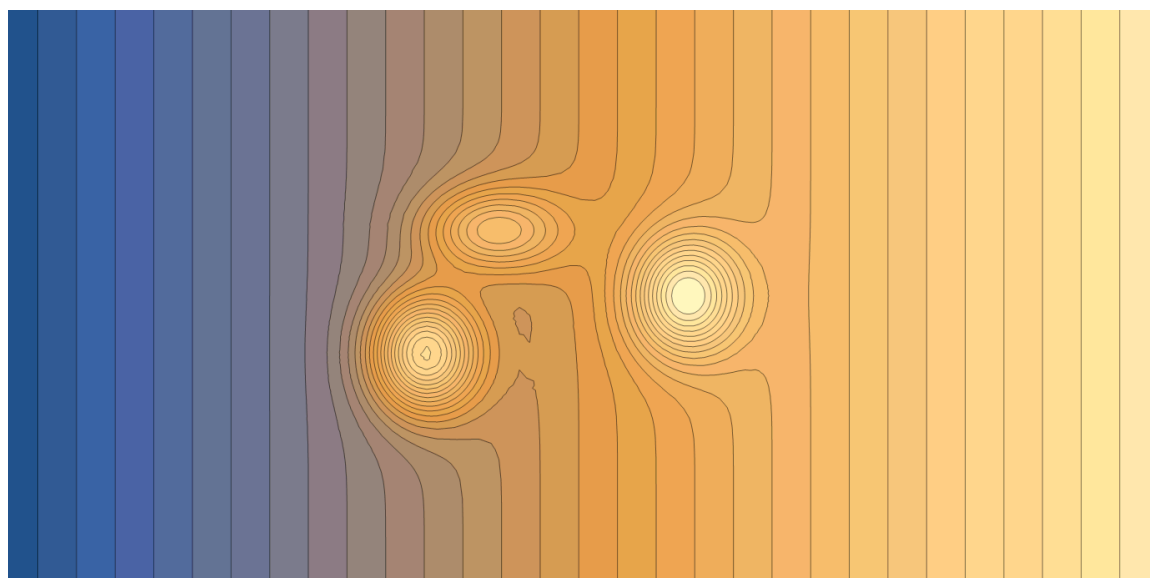

Figure 3. Regions with high entropic gradients towards higher dimensional complexity, which allow for self-organizing criticalities

\subsection{Dependancy on initial state and dynamics}

Whether or not the state of a system actually reaches such a self-organizing criticality depends on its initial state, as shown in Figure 4.

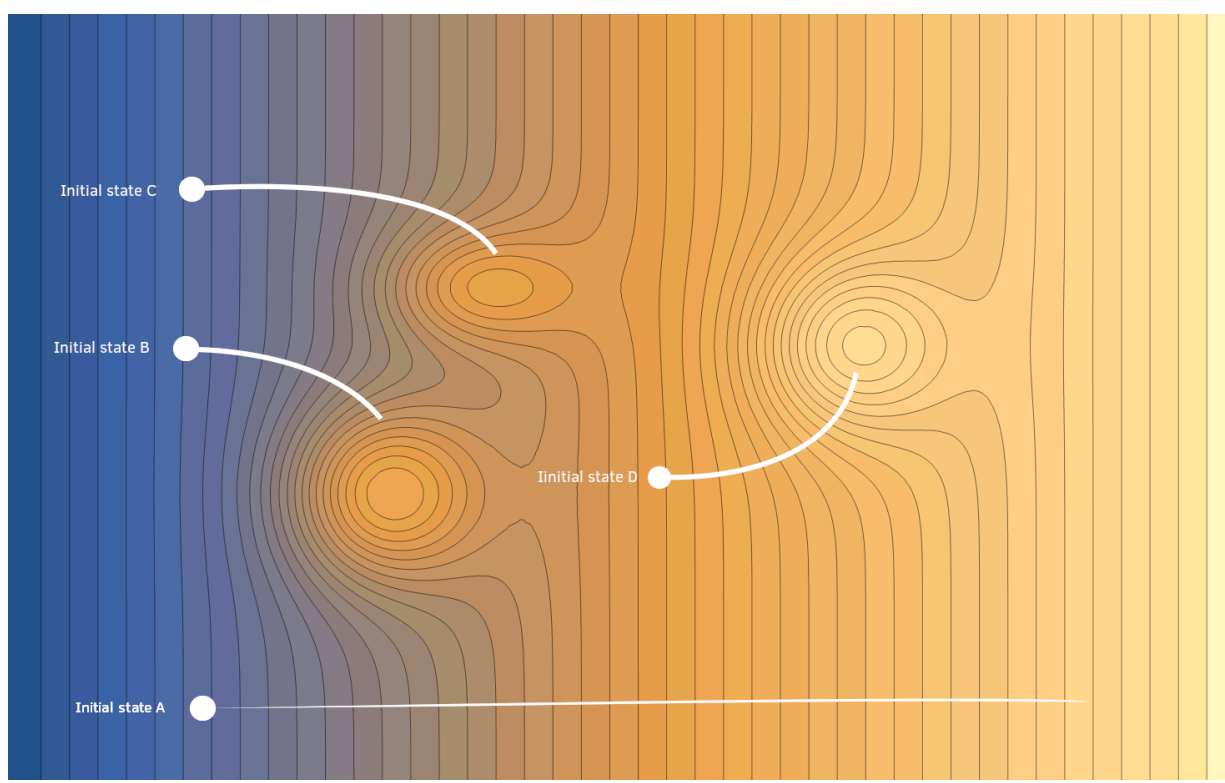

Figure 4. The system maximizes its entropy production relative to its initial state

But it also depends on the dynamics of the state-space. If you consider the (open) system of a tree, its state-space changes over time, because of changes in the regional ecosystem and in the local weather-conditions, for example. If there is a period of severe drought, existing local maxima can bifurcate away.

\subsection{Higher-level maximization}

The example of the system of a tree is a good example of a fractal superstructure. The dynamics of the state-space of this tree depends on at least its surrounding ecosystem and the weather. But these are also nonlinear dynamic systems, with highly intricate state-spaces with many local maxima of entropy production of their own. And towards 
the micro-level all the cells of the tree (and in between its leaves and its seeds) can be seen as subsystems with their own dynamics and dynamic state-spaces. So, ultimately, the choice of what you consider a (sub)system is strictly arbitrary, as these dynamics are all related. The same goes for our economic system, or a technological system. All these systems can be seen as superstructures, hierarchical ensembles with some fractal interdependency of non-linear dynamics, from micro to macro-level.

And from the same statistical inference as described above, eventually all these related dynamics will tend towards an optimal distribution of local maxima of entropy production, throughout the total ensemble.

\section{Results}

In this article it is demonstrated that the maximization of entropy production that we observe in many domains actually follows from the same statistical inference as that of the maximization of entropy itself (i.e. the second law of thermodynamics). Entropic gradients in complex dynamic systems can be multidimensional, and a system will always follow the steepest direction, either into states with higher dimensional complexity if available, or otherwise within the same dimensionality. We have introduced the concept of a super-statespace (for systems with dynamic statespaces) with zero dimensions (resulting in a single solution for a statespace) or more dimensions (enabling dynamic statespaces). We can then generalize the entropic gradient of a system with a zero-dimensional super-statespace to a special case of entropic gradients of systems with super-statespaces of any dimensionality, as a means to formalize the general validity of the maximization of entropy production.

\section{Discussion}

The demonstration of the general validity of the MEPP has impact on our understanding of the world around us, as it applies to many domains. Next to the obvious example of meteorology, it explains the 'autopoiesis' of life and other growing structures. It can even help explain many macro-trends in our growing financial systems, technological domains, our socio-economic domains, and administrative burocracies. Also, many trends in the IT-revolution and globalization can be better understood from this perspective as these trends have yielded super-statespaces of increasing dimensionality of structural complexity and dynamics (with many wanted and unwanted consequences). Ultimately this understanding may also help in developing much more effective and empirically based policies at many levels, as every policy is then actually a matter of increasing or decreasing complexity in some subregion, to keep them within some thresholds that are aligned with some normative valuation framework, for example. It suggests that to affect certain behavioral dynamics, it is much more effective to alter the facilitating structural complexity, than to try to regulate this behavior.

Funding: This research received no external funding

Conflicts of Interest: The authors declare no conflict of interest.

\section{Abbreviations}

The following abbreviations are used in this manuscript:

MEPP Maximum Entropy Production Principle

\section{References}

1. Dewar, R. (2003). Information theory explanation of the fluctuation theorem, maximum entropy production and self-organized criticality in non-equilibrium stationary states. Journal of Physics A: Mathematical and General, 36(3):631-641.

2. Dewar, R. (2005). Maximum entropy production and non-equilibrium statistical mechanics. In Kleidon A., L. R., editor, Non-equilibrium Thermodynamics and the Production of Entropy. Understanding Complex Systems.., chapter 4. Springer.

3. Dobovišek, A., Vitas, M., Brumen, M., and Fajmut, A. (2017). Energy conservation and maximal entropy production in enzyme reactions. BioSystems, 158:47-56. 
4. Garrett, T., Grasselli, M., and Keen, S. (2020). Past production constrains current energy demands: persistent scaling in global energy consumption and implications for climate change mitigation. arXiv econ.GN 2006.03718

5. Hill, A. (1990). Entropy production as the selection rule between different growth morphologies. Nature, 348(6300):426-428.

6. Kleidon, A. (2010). A basic introduction to the thermodynamics of the Earth system far from equilibrium and maximum entropy production.

7. Martyushev, L. M. (2010). The maximum entropy production principle: Two basic questions. Philosophical Transactions of the Royal Society B: Biological Sciences, 365(1545):1333-1334.

8. Martyushev, L. M. and Seleznev, V. D. (2014). The restrictions of the maximum entropy production principle. Physica A: Statistical Mechanics and its Applications, 410:17-21.

9. Ozawa, H., Ohmura, A., Lorenz, R. D., and Pujol, T. (2003). The second law of thermodynamics and the global climate system: A review of the maximum entropy production principle. Reviews of Geophysics, 41(4):1018.

10. Paltridge, G. (2005). Stumbling into the mep racket: An historical perspective. In Kleidon A., L. R., editor, Non-equilibrium Thermodynamics and the Production of Entropy. Understanding Complex Systems., chapter 3. Springer.

11. Paltridge, G. W. (1975). Global dynamics and climate - a system of minimum entropy exchange. Quarterly Journal of the Royal Meteorological Society, 101(429):475-484.

12. Prigogine, I. and Nicolis, G. (1971). Biological order, structure and instabilities. Quarterly Reviews of Biophysics, 4(2-3):107.

13. Ramstead, M. J. D., Badcock, P. B., and Friston, K. J. (2017). Answering Schrödinger's question: A free-energy formulation. Physics of Life Reviews, 24:1-16. 